\title{
Psychiatric Drug Prescription and Temporal Associations with a First Diagnosis of Gambling Disorder-Results from a National Register Study
}

\author{
Carolina Widinghoff ${ }^{1,2}$ (D) Jonas Berge ${ }^{1,2} \cdot$ Anders Hakansson $^{1,2}$
}

Accepted: 23 August 2021 / Published online: 7 September 2021

(c) The Author(s) 2021

\begin{abstract}
Psychiatric comorbidity is common in gambling disorder (GD), but there are few studies on larger nationwide samples of treatment-seeking patients. Also, temporal associations between GD and other psychiatric disorders are often difficult to study. To address the prevalence and the temporal associations of prescriptions for psychiatric disorders - both in specialized care and primary care - in patients with a GD diagnosis (ICD-10 F63.0). Data was derived from national health registers in Sweden. All patients who were diagnosed with GD in specialized health care in 2005-2016 were included and run against the nationwide database on prescription of pharmaceuticals aimed for psychiatric disorders $(n=2018)$. Prevalence of psychiatric drug prescription was used as a proxy for psychiatric comorbidity and studied for two 2-year periods (period 1 and 2) prior to GD and one 2-year period (period 3) after the diagnosis. Controlling for gender, age, and time periods, for eight drug categories (anti- epileptics, anti-psychotics, benzodiazepine derivatives, anxiolytics, hypnotics, anti- depressants and drugs used in addictive disorders), significant increases in drug prescription were seen. For central stimulants, a significant increase was seen upon receiving the GD diagnosis (from period 2 to 3 ), and for benzodiazepines, an increase was seen prior to the GD diagnosis (from period 1 to 2), but not upon diagnosis (from period 2 to 3). Psychiatric comorbidity in GD is common. Drug prescription for psychiatric problems increased markedly in the years temporarily associated with a first diagnosis of GD. The findings may call for early screening for problem gambling in patients with treatment contacts for increasingly poor mental health.
\end{abstract}

Keywords Gambling disorder · Psychiatric comorbidity · Addiction medicine · Psychiatric drug prescription $\cdot$ Register study

Carolina Widinghoff

carolina.widinghoff@med.lu.se

1 Department of Clinical Sciences Lund, Psychiatry, Faculty of Medicine, Lund University, S-22100 Lund, Sweden

2 Gambling Disorder Unit, Region Skåne, S-20502 Malmö, Sweden 


\section{Background}

Gambling disorder (GD), classified as a non-substance-related addictive disorder in the Diagnostic and statistical manual of mental disorders (5th ed.) (American Psychiatric Association, 2013), is associated with an elevated risk of indebtedness, interpersonal violence, and suicide, and the psychiatric comorbidity is high (Hakansson \& Widinghoff, 2020b; Moghaddam et al., 2015; Petry et al., 2005; Ronzitti et al., 2016). Yet, there is a lack of studies describing treatment-seeking patients with GD and current psychiatric disorders (Dowling et al., 2015b; Hakansson et al., 2018). It is known that GD has a highly varying course, where movement in and out of gambling severity levels is common (LaPlante et al., 2008), and about one third of the patients are reported to recover naturally (Slutske, 2006), making it methodologically challenging to study the temporal associations between problematic gambling and other psychiatric conditions. In the literature, common pathophysiological factors between GD and several other disorders are described, and certain pharmacological compounds appear to be more effective in subgroups of GD patients (Di Nicola et al., 2020a). Greater knowledge about gambling problems and psychiatric comorbidity could contribute to a better understanding of the appearance of these disorders, possibly coming up to more effective preventive actions and personalized treatment.

In Sweden, online gambling has been a growing problem for years, and the proportion of people with gambling problems seems to increase in parts of the population (Abbott et al., 2018; Hakansson \& Widinghoff, 2020b). According to the latest estimations, point prevalence of GD is about $0.6 \%$ in the adult population, but lifetime prevalence is considerably higher (Abbott et al., 2017). In parallel, it is known that few people with gambling problems seek help (Gainsbury et al., 2014; Suurvali et al., 2009), and in another recent study, a large proportion of the general population reported that they would not refer a friend with hypothetical GD to professional treatment, but rather to non-professional patient organizations (Ford \& Hakansson, 2020). The topic is a public health issue, and it is a challenge for healthcare and society to prevent gambling problems, and to reach people in need of addressed treatment (Wardle et al., 2019).

Recent studies from the present setting have shown that psychological distress is high among people with high risk or problem gambling, especially in women (Hakansson \& Widinghoff, 2020b). In a general population study, $61.8 \%$ of people with gambling problems reported having had any other psychiatric disorder compared to $46.9 \%$ of the controls, with at least a double risk of a lifetime psychiatric disorder among problem gamblers (Sundqvist \& Rosendahl, 2019). Despite the well-known fact that psychiatric comorbidity is common in GD patients, there is a paucity of research on total population data, as most studies have been conducted in limited clinical settings (Dowling et al., 2015b). However, a comprehensive study describing patients who had been diagnosed with GD in Swedish specialized health care, with respect to treatment uptake and co-occurring psychiatric diagnoses, also made in specialized in- or outpatient care, was recently published (Hakansson et al., 2018). According to the results, $73 \%$ of the patients with GD in specialized health care had at least one more psychiatric diagnosis, and even though treatment uptake for GD was increasing the last years, there were relatively few patients in treatment compared to the estimated number of problem gamblers in the general population, pointing to the need of identifying these 
individuals to provide support and treatment at an earlier stage. Some of the methodological limitations in the study were the lack of longitudinal perspective with respect to the comorbidity, and omission of possible co-occurring diagnoses made in primary health care since those were not available in the register used. Therefore, this study was conducted on the same data material consisting of a population diagnosed with GD in specialized health care, investigating three sequential time periods and with prescription of psychiatric drugs as proxies for psychiatric comorbidity, since the national register of prescribed drugs covers prescription from primary care as well.

\section{Aims}

Comorbidity between GD and a number of other psychiatric conditions is common but, as discussed above, there are relatively few studies describing GD in the context of cooccurring psychiatric diagnoses in the health care system (Hakansson et al., 2018). The aim of the present study was to investigate the prescription of psychiatric drugs prior to and following a first diagnosis of GD, to assess temporal associations between a first diagnosis of GD psychiatric comorbidity.

\section{Methods}

\section{Participants}

The primary inclusion criterion was having received a GD diagnosis in Swedish specialized health care. During the studied period, from the first of July 2005 to the 31st of December 2016, 2172 patients received a GD diagnosis for the first time. Due to the risk of incorrectly made diagnoses in young patients, those under 18 years of age were excluded $(n=73)$. This was done for two reasons: first, gambling is not allowed for underage persons $(<18$ years) in Sweden; hence, actual GD is probably not very likely in younger ages. Secondly, for linguistic reasons, these patients may have been suffering from gaming - and not gambling — disorder, since the terminology in Swedish could potentially be confused. Data was missing for participants who received their GD diagnoses too close to the time limits of the study. The number of included participants in each period was 1786 (missing data in 313 cases) in period 1, with corresponding numbers of 1904 (missing data in 195 cases) in period 2, and 1670 (missing data in 429) in period 3. The total number of included participants was 2018.

\section{Registers}

Data was collected from two registers: the Swedish National Patient Register (NPR), including all episodes of specialized medical in- and out-patient treatment for both psychiatric and somatic care, and from the Swedish Prescribed Drug register (PDR), covering all medications issued on prescription by pharmacies. The NPR was established in 1964 and is reported to have a high validity, fully covering in-patient care since 1987 (Ludvigsson et al., 2011), and coverage of out-patient care has increased successively to about $97 \%$ of primary diagnoses in 2018 (Swedish National Board of Welfare, 2020a), but the register is limited to specialized care and including no 
information from primary care. The PDR on the other hand covers all prescribed drugs since the first of July 2005, regardless of prescriber employment, meaning that this register could contribute with indirect information about healthcare given also from primary care health centers. The main inclusion criterion was having received a pathological gambling diagnosis (F63.0 in the ICD-10, the International Classification of Diseases and related Health Problems, 10th revision) (World Health Organization, 2004), corresponding to the diagnosis GD in the Diagnostic and statistical manual of mental disorders (5th ed.) (American Psychiatric Association, 2013) in specialized health care between 2005 and 2016.

"Prescription" was defined as drugs having been prescribed by physicians and dispensed from pharmacies. For reference regarding prescription of psychiatric drugs in the general Swedish population, we used the public statistic database by the website of the National Board of Health and Welfare (2020b), covering all prescribed drugs during 2006-2019. We limited the search to the ATC codes in question for this study (see below under "Drugs"), the most suitable selectable age group (20-85 + years), all national regions, both genders, the years 2006-2016, and number of patients divided by 1000 inhabitants (Swedish National Board of Welfare, 2020b).

\section{Drugs}

The psychiatric drugs were categorized in eight groups according to the international Anatomical Therapeutic Chemical (ATC) Classification System (World Health Organization, 2020): antiepileptics (N03), antipsychotics (N05A), benzodiazepine derivatives (N05BA), anxiolytics (N05BB and N05BC), hypnotics (N05C), antidepressants (N06A), psychostimulants (N06B), and drugs used in addictive disorders (N07B).

The above-listed drug categories are being prescribed for several different psychiatric, and in some cases also somatic, conditions. Antiepileptic drugs have several approved indications, and off-label areas of use, beyond actual epilepsy. For instance, they are used to treat chronic pain, acute bipolar depression, posttraumatic stress disorder, and affective dysregulation and behavioral dyscontrol domains of borderline personality disorder (Naguy \& Al-Enezi, 2019; Swedish National Board of Welfare, 2018). Antipsychotics are approved for schizophrenia, bipolar disorder, and, for some drugs, depression, and parallel to antiepileptics, they are used addressing multiple other psychiatric diagnoses. Since the introduction of atypical antipsychotic drugs in the 1990s, broad off-label use is reported, such as in generalized anxiety disorder, obsessive compulsive disorder, eating disorders, personality disorders, and posttraumatic stress disorder (Maglione et al., 2011). Benzodiazepines are being prescribed in almost all psychiatric conditions, usually addressing anxiety or sleeping problems, and have strong evidence-based anxiolytic and anticonvulsant effects (Dell'Osso et al., 2015; Wick, 2013). The category anxiolytics here refers to the drug hydroxyzine, a first-generation antihistamine approved for treatment of urticaria, itching, and anxiety (LiverTox, 2017). In accordance with the ATC system, hypnotics include propiomazine, benzodiazepine derivatives, and benzodiazepine like hypnotic drugs. Antidepressants of different types are approved for a wide range of psychiatric and somatic conditions; major depression, anxiety disorder, obsessive-compulsive disorder, chronic pain, sleep disorders, bulimia, posttraumatic stress disorder, nocturnal enuresis in children, and incontinence in women (The Swedish Association of the Pharmaceutical Industry, 
2020). Psychostimulants are prescribed to treat attention deficits, mainly in children and adolescents (Swedish Medical Products Agency, 2016). The category drugs used in addiction treatments include medications used to treat dependence of nicotine, alcohol, and opioids (World Health Organization, 2020).

\section{Time Periods}

We defined three periods of observation based on the date of the first GD diagnosis in the (NPR). The first period was 4 to 2 years prior to the diagnosis. The second period was 2-0 years prior to the diagnosis, and the third period was 2 years following the diagnosis. Each study participant thus had a maximum of three possible observation periods. Complete data for all three periods required coverage of prescriptions 4 years before GD diagnosis and 2 years after. GD diagnosis was made at the first day of period 3. For an observation period to be considered valid, the whole period had to be covered by the data from the PDR. Because the data from the PDR starts at July 1, 2005, and the end date for our study is December 31, 2016, some study participants will have one or more observation periods considered invalid. For instance, if a particular individual received the GD diagnosis in September 2008, the first period is considered invalid because the PDR does not cover the entire period of September 2004 to September 2006, but the second and third periods are considered valid because they are entirely covered in the PDR data. Similarly, if an individual received the GD diagnosis in April 2015, the first two periods are considered valid, but the third period is considered invalid because the PDR data does not cover the entire period until April 2017.

Having defined the periods and which periods are valid for each individual, we then created binary variables for each of the eight drugs for each of the three periods. If an individual had been prescribed at least one drug from each class of drugs a given period, then the individual was considered as being in treatment with that drug in that period.

\section{Statistical Analysis}

We used a repeated-measures design under a generalized mixed-model regression framework, using a logit link and binary distribution assumption, to assess the association between GD diagnosis and change in prescription rates for each of the drug classes, where the dichotomous treatment variables for each of the drug classes constituted the repeated measurements. The first variable in the model was time, defined as the three 2 -year periods as described above. Inclusion of this variable in the models provided an estimate of the change in treatment for the study population over time. We next added the variable diagnosis, which was defined as 0 for the first two periods and 1 for the third period. The combination of these two variables enabled us to assess both the linear association between time and treatment and the deviation from this association following the GD diagnosis. The variable diagnosis thus constituted the main variable of interest for the purposes of the present study.

Chronological year at the time of the GD diagnosis, participant age at the time of the diagnosis, and participant gender were included as covariates in the model. The generalized mixed model analysis allowed the estimation of random intercepts, thereby taking the violation of the independence of observation assumption required in the standard regression model framework. 


\section{Procedure}

It was investigated which psychiatric drugs the participants had been prescribed before and after the first GD diagnosis. Changes in proportions of participants with prescriptions of each drug group were estimated, controlling for time periods, possible impact of GD diagnosis, years passed, age, and gender.

The present study is based on Swedish National registers and approved by the Regional Ethics Committee, Lund, Sweden (file number 2016/1104). It was conducted in accordance with the Swedish ethics legislations, and no informed consent procedure was required, since the patient material consists of non-identified data from national register units.

All statistical analysis were performed in SPSS version 26 (IBM Corp., 2019) and R version 3.5.3 (R Core Team, 2019).

\section{Results}

Among the 2018 included participants, the mean age was 36.5 years with a range of 18-83 (standard deviation 11.9), and $22.6 \%(n=474)$ were female. GD diagnoses were established within in-treatment episodes in $29.2 \%(n=591)$ of the cases, within out-patient treatment in $85.7 \%(n=1729)$ of the cases, and $15.0 \%(n=302)$ had been diagnosed with GD in both in-patient and out-patient treatment. A total of $46.6 \%(n=938)$ had received GD as a primary diagnosis.

Proportions of patients with prescriptions of each drug category, according to ATC codes, for each of the three periods are shown in Table 1. In summary, there is an increase for every drug category between periods. Compared to the general population (Swedish National Board of Welfare, 2020b), the yearly prescriptions were higher for each drug group during the studied period (corresponding percentages of dispensed drugs in the general population in parenthesis): antiepileptics $8.0-12.4 \%$ (1.9-3.0\%), antipsychotics $11.4-21.0 \%$ (2.0-2.2\%), benzodiazepines $10.1-15.4 \%$ (4.5-5.0\%), hydroxyzine

Table. 1 Proportions of patients with prescriptions of each drug category (according to ATC codes) during three different periods

\begin{tabular}{llllll}
\hline Drug group & ATC & Period 1 & Period 2 $^{\mathrm{b}}$ & Period 3 $^{\mathrm{c}}$ & Total $^{\mathrm{d}}$ \\
\hline & code & $\%(n)$ & $\%(n)$ & $\%(n)$ & $\%(n)$ \\
Antiepileptics & N03 & $8.0(143)$ & $10.6(201)$ & $12.4(207)$ & $15.6(314)$ \\
Antipsychotics & N05A & $11.4(204)$ & $15.7(298)$ & $21.0(351)$ & $23.2(468)$ \\
Benzodiazepine derivatives & N05BA & $10.1(180)$ & $13.9(265)$ & $15.4(257)$ & $20.9(422)$ \\
Anxiolytics & N05BB, N05BC & $9.4(168)$ & $14.1(268)$ & $17.0(284)$ & $26.5(534)$ \\
Hypnotics & N05C & $20.5(366)$ & $27.2(518)$ & $36.8(614)$ & $42.0(848)$ \\
Antidepressants & N06A & $31.9(570)$ & $42.9(816)$ & $53.8(898)$ & $59.7(1204)$ \\
Psychostimulants & N06B & $5.4(97)$ & $5.7(109)$ & $7.1(118)$ & $8.5(172)$ \\
Drugs used in addictive disorders & N07 & $2.5(44)$ & $4.7(89)$ & $10.5(175)$ & $11.8(238)$ \\
\hline
\end{tabular}

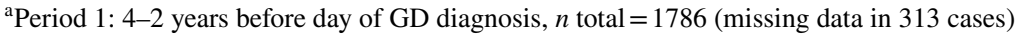

${ }^{\mathrm{b}}$ Period 2: 2-0 years before day of GD diagnosis, $n$ total $=1904$ (missing data in 195 cases)

${ }^{\mathrm{c}}$ Period 3: Day of GD diagnosis and following 2 years, $n$ total $=1670$ (missing data in 429 cases)

${ }^{\mathrm{d}}$ Total $n=2018$, valid percentages 
9.4-17.0\% (1.9-3.0\%), hypnotics 20.5-36.8\% (10.4-10.5\%), antidepressants 31.9-53.8\% (10.3-12.1\%), psychostimulants $5.4-7.1 \%(0.13-0.79 \%)$, and drugs used in addictive disorders $2.5-10.5 \%(0.5-0.7 \%)$.

The odds ratios of each drug group in the three time periods controlling for period, possible impact of GD diagnosis, years passed, age, and gender are shown in Table 2 and Diagram 1. The variable "period" shows significant increases proportion of prescriptions between periods 1 and 2 and between periods 2 and 3, for each category; antiepileptics (OR 3.86, 95\% CI 2.39-6.24, $p<0.001$ ), antipsychotics (OR 4.39, 95\% CI 2.87-6.71, $p<0.001$ ), benzodiazepine derivatives (OR 2.96, 95\% CI 2.01-4.36, $p<0.001$ ), anxiolytics (OR 1.84, 95\% CI 1.44-2.34, $p<0.001$ ), hypnotics (OR 2.13, 95\% CI 1.68-2.69, $p<0.001$ ), antidepressants (OR 2.55, 95\% CI 2.09-3.11, $p<0.001$ ), and drugs used in addictive disorders (OR 4.64, 95\% CI 2.56-8.42, $p<0.001$ ), except psychostimulants (OR 1.94, 95\% CI $0.94-3.97, p=0.071)$. "GD diagnosis" represents the possible impact of the GD diagnosis, by showing the change in odds ratio for period 3 compared to expected linear progress after period 1 and 2. Significant changes are seen for benzodiazepines, with a significant decrease in prescription after GD diagnosis (OR 0.34, 95\% CI $0.18-0.64, p=0.001$ ) and for psychostimulants, with a significant increase for the corresponding period (OR 3.85, 95\% CI 1.12-13.27, $p=0.033)$. "Year" represents the change in frequency of prescriptions for each year, showing a significant decrease for antidepressants (OR 0.91, 95\% CI $0.87-0.96, p<0.001$ ) and hypnotics (OR $0.87,95 \%$ CI $0.82-0.92, p<0.001$ ). The variable "age" represents the average change in frequencies of prescription for each year of age, showing small but significant increases for benzodiazepines, (OR 1.47, 95\% CI 1.14-1.90, $p=0.003$ ), hypnotics (OR 1.84, 95\% CI 1.57-2.17, $p<0.001$ ), and antidepressants (OR $1.45,95 \%$ CI $1.27-1.64, p<0.001)$. In participants with female gender, it was significantly more common with prescriptions for antiepileptics (OR 2.81, 95\% CI 1.25-6.32, $p=0.012$ ), anxiolytics (OR 2.46, 95\% CI 1.79-3.38, $p<0.001$ ), hypnotics (OR 4.71, 95\% CI 2.98-7.45, $p<0.001$ ), and antidepressants (OR 5.2, 95\% CI 3.58-7.54, $p<0.001$ ).

\section{Discussion}

The aim of this study was to investigate the prescription of psychiatric drugs in connection with the establishment of GD diagnoses made in specialized medical care, in order to describe the psychiatric comorbidity. As far as the authors of this study know, there are few previous studies investigating pharmacological treatment temporarily associated with GD diagnoses, showing the need of further knowledge about comorbidity from a new perspective in this research area. By studying drugs as proxies for psychiatric comorbidity during these three periods prior to and following GD diagnoses, we can present new data revealing an extensive comorbidity and, likely, major suffering, in this group of patients. GD patients with further psychopathology have a poorer prognosis (Muller et al., 2017) and are in need of a certain psychosocial and pharmacological assessment (Di Nicola et al., 2020a; Dowling et al., 2015a). This study might contribute, together with previous literature describing GD-related psychiatric comorbidity and effects of specific psychiatric drugs on GD and related disorders, to new hypotheses for optimizing GD treatment and further research aiming to find algorithms (Di Nicola et al. 2020a) leading to patient-tailored psychosocial and pharmacological treatment programs.

The overall result of this study was a high and increasing prescription of psychiatric drugs among the participants during the studied period and compared to the 


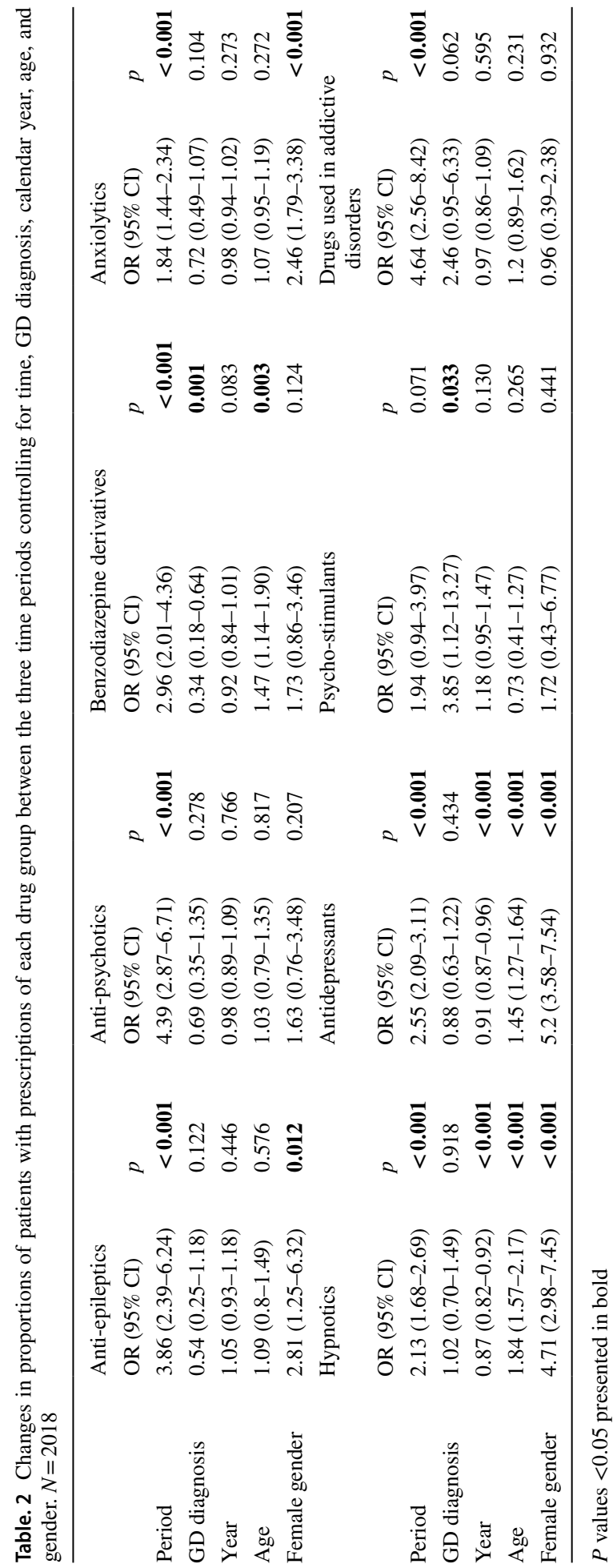



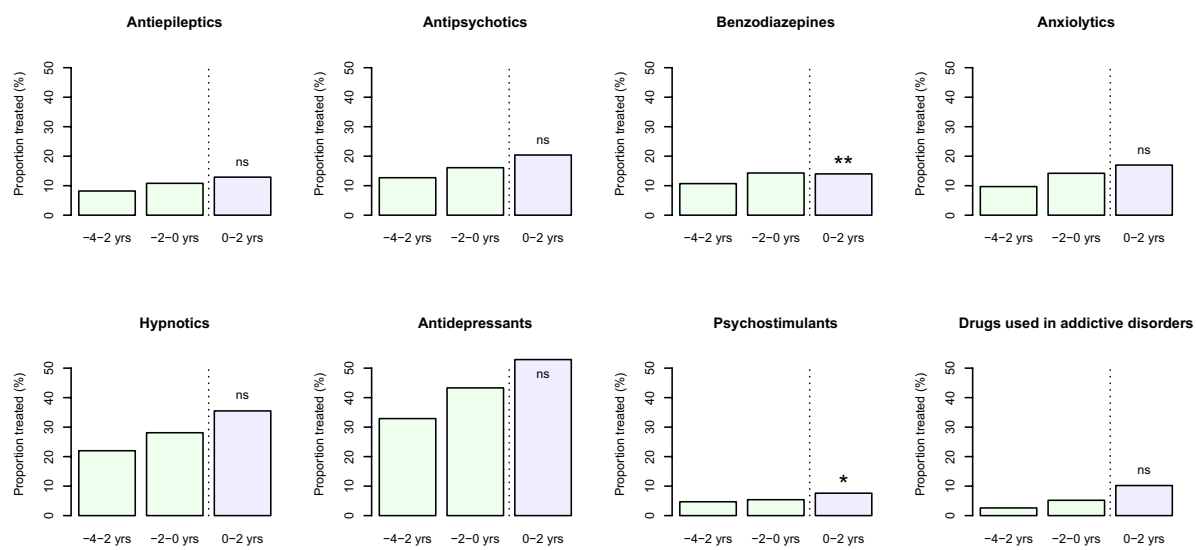

Diagram 1 Changes in proportions of patients with prescriptions of each drug group between the three time periods. The test for statistical significance reported in the figures refers to the change in linear trends in period 3, controlling for time, calendar year, age, and gender

general population yearly prescriptions were remarkably high for each drug group (Swedish National Board of Welfare, 2020b). According to regression analysis, there were increases between periods for all drug groups except for psychostimulants, which increased after GD diagnosis. Trends of increasing anxiety and depression have been reported in Sweden for several years and should be interpreted as a widespread issue with a wide range of reasons (Swedish National Board of Welfare, 2019), but our results indicate a markedly poor mental health in the studied patient group. Regarding psychiatric drugs as proxies for psychiatric comorbidity in this context, the results point toward worsened mental health during the life periods when the GD diagnoses were established, since close to $60 \%$ of the participants in the present study were prescribed antidepressants, compared to around $12 \%$ of the general population. General trends of increased prescription of both antidepressants and antiepileptics, the latter being prescribed for varying conditions in need of a mood stabilizing, sedative, or analgesic effect (Goodman \& Brett, 2017; Naguy \& Al-Enezi, 2019; Welfare, 2018), have been observed, but among the study participants, the increase, as well as the prescription in total, appears to be substantially higher than in the general population (Swedish National Board of Welfare, 2019, 2020b). This is intuitively understandable, considering the well-known facts that several psychiatric diagnoses are associated with GD, and that the condition itself involves several serious consequences causing mental distress (American Psychiatric Association, 2013; Dowling et al., 2015b). Parts of the pathobiological mechanisms behind this comorbidity are explained in previous studies, reporting that impaired dopaminergic and noradrenergic neurotransmission systems contribute to emotional vulnerability and addictive behavior, which may in turn lead to compensatory responses increasing the risk of maintained affective instability and substance or behavioral addiction (Di Nicola et al., 2020b). Without overstating the meaning of associations studied here, it can be concluded that patients receiving GD diagnoses in specialized health care were experiencing pronounced mental distress, leading to prescription of antidepressants to a large and increasing extent years before and after establishment of GD diagnoses.

There is so far no pharmacological drug approved by the Swedish Medical Products Agency with an indication for GD. Hence, the pharmaceuticals reported here are most likely prescribed for other diagnoses and can be contemplated as proxies for the 
co-occurring psychiatric conditions. However, there are studies suggesting that pharmacological treatment may have effect on GD, especially if chosen with respect to possible co-occurring psychiatric diagnoses Di Nicola et al., 2020a, Di Nicola et al., 2014; Yip \& Potenza, 2014). The opioid antagonist naltrexone is reported being the most widely studied drug in GD treatment and may be effective, particularly in patients with parallel substance use disorder or heritability for alcoholism (Chamberlain \& Grant, 2019; Goslar et al., 2019; Victorri-Vigneau et al., 2018). Drugs used in addictive disorders were prescribed to $2.5-10.5 \%$ of the study participants, with a corresponding number around $0.5 \%$ of the general population (Swedish National Board of Welfare, 2020b). Considering the wellknown comorbidity between GD and other addictive syndromes (Dowling et al., 2015b), the high prescription of these drugs in the studied patient group is not surprising. Prescription in period 1 and 2 indicates that up to almost $5 \%$ of the study participants were in pharmacological treatment for an addictive disorder during the years foregoing the establishment of GD diagnoses. It is possible that there was an on-going, undiscovered GD during this period, or that substance use disorders converted or widened to GD, as alternating between different behavioral or substance addictions is a previously described phenomenon (LaPlante et al., 2008). There was a trend toward a particular increase in prescription temporarily associated with the establishment of GD diagnosis in period $3(p=0.062)$ which could be connected to a larger possibility of discovering and/or treating substance use disorder after GD had been discovered.

We specifically investigated changes in prescription in connection with establishment of GD diagnoses, by analyzing possible breaks of the linear prescription trends. These measures are complex to interpret, considering the methodological challenges concerning chronology between actual gambling problems and GD diagnoses, and varying indications for psychiatric drugs, but may still contribute to valuable knowledge about clinical treatment of these patients. For all the investigated drug groups, the prescription rates were generally high and increasing and, except for benzodiazepines and psychostimulants, there were no significant changes connected to GD diagnoses. This might indicate that the psychiatric health in the studied population was often poor years ahead of the GD diagnoses, likely in a reciprocal and mutually reinforcing relationship between mental health issues and gambling behavior. Concerning benzodiazepines, a significant decrease of the increasing linear trend over the time periods was seen, resulting in comparable figures for the last two time periods, probably due to reluctance of prescribing this type of medication as benzodiazepines are recommended to be prescribed with caution to patients with substance abuse (Dell'Osso et al., 2015). Another interpretation of this, except that doctors may have been less prone to prescribe benzodiazepines to patients with known addiction problems in the current study, could be that some of the participants' anxiety problems and hence the need of benzodiazepines actually flattened out after establishment of GD diagnoses and initiation of other treatment (e.g., cognitive behavioral therapy or motivational interviewing), as recommended (Di Nicola et al., 2020a; Yip \& Potenza, 2014). Benzodiazepines are especially effective in anxiety treatment, but are also highly addictive, and appear to be indicators of the severity of the psychiatric comorbidity, as the use is higher in patients with dual diagnoses and associated with higher scores of affective symptoms, lower scores for quality of life, and elevated risks of progression to abuse (Dell'Osso et al., 2015). Concerning the prescription of benzodiazepines in the general population, there was no increase during the studied period, but higher rates in older patients (Swedish National Board of Welfare, 2020b), which is in line with our results in controlled analysis. According to previous literature, long-term use is more common among older people, but there seems to be an overall declining trend in a number of contexts, probably due to awareness of the addictive 
potential, and the availability of the new antidepressants (Balon, 2013; Kurko et al., 2018; Swedish National Board of Welfare, 2019; Torres-Bondia et al., 2020).

As in the case with benzodiazepines, stimulants are highly potent drugs with addictive potential, and there is a known misuse and diversion (Clemow, 2017; Dell'Osso et al., 2015). An increase in prescription of psychostimulants was seen following GD diagnoses, hypothetically due to increased probability of detecting and initiating treatment for attention deficit hyperactivity disorder (ADHD) in connection with establishment of GD diagnoses, considering the known comorbidity between the two disorders. In the general population there was an annual prescription below $1 \%$ but increasing from 0.13 to 0.79 and markedly higher among children under the age of 18, during the studied period (Swedish Medical Products Agency, 2016; Swedish National Board of Welfare, 2020b). Psychostimulants as a drug group call for certain attention and deliberation in a gambling context, for several reasons. There are pronounced associations between GD, impulsivity, and ADHD (Mitchell \& Potenza, 2014), and patients with both ADHD and GD may be in particular need of treatment but are also in high risk for negative consequences of stimulant medication (Zack \& Poulos, 2004). The scientific evidence for medical treatment of patients with ADHD and comorbid addictive disorders is still limited (Crunelle et al., 2018; Perugi et al., 2019), and it has been suggested that these drug types work partly through the same neurological pathways as gambling, causing excitement by increased release of dopamine in nucleus accumbens, which may prime gambling behavior (Zack \& Poulos, 2009). In a placebo-designed study by Zack and Poulos (2004), investigating the effects of a low oral dose of dexamphetamine $(30 \mathrm{mg})$, problematic gamblers experienced a significantly higher desire to gamble and lower confidence to resist gambling compared to control groups. Groen and colleagues (2013) stated in a review that no definite conclusions could be drawn so far concerning the effects of methylphenidate on gambling behavior, due to the small number of studies and inconsistent findings. In parallel, stimulant treatment for ADHD in patients with substance use disorders is controversial concerning both efficacy and safety (Crunelle et al., 2018). ADHD predisposes to substance use disorders through various mechanisms, probably due to self-medication and impulsivity (Cunill et al., 2015), similar to the biopsychological factors posing the risk for gambling problems, and crosssensitization by stress, drugs, and gambling behavior has been suggested as an explanation for this comorbidity (Hellberg et al., 2019). There are studies suggesting that stimulant treatment for ADHD may have a long-term protective effect against substance abuse (Biederman, 2003; Chang et al., 2014), but there are also studies showing no such effect (Humphreys et al., 2013) and alarming research reporting ADHD medication abuse among adolescents rising along with the prescription (Setlik et al., 2009). Experts in the area have stated that stimulants may play an important role for treatment of co-existing ADHD and SUD in large parts of the adult patient group and call for further research to identify relevant subgroups (Perugi et al., 2019).

As sleep disorders are common in patients experiencing mental distress (Proctor \& Bianchi, 2012), it is not surprising that a total of $42.0 \%$ of the patients in the studied group were prescribed hypnotics, with a significant increase between periods (20.5-36.8\%). During the studied period, the prescription of hypnotics in the general population was about $10 \%$ and stable over time (Swedish National Board of Welfare, 2020b). In a study by Parhami and colleagues (2013), two thirds of pathological gamblers reported sleep complaints, and sleeping problems can be extreme during periods of intensified gambling, crisis, or withdrawal (Parhami et al., 2013). Sleep disturbances occur in a variety of psychiatric conditions and the fact that establishment of GD diagnosis in period 3 did not seem to increase the prescriptions of hypnotics significantly in controlled analyses in this study might be 
explained by comorbidity preceding GD, or that the gambling problems were clinically occurring before the actual establishment of GD diagnosis. Of course, sleeping problems might also have been present in various degrees in the studied patients without medical treatment addressing them. Despite not coming into sight in this study, likely due to methodologic reasons, previous literature has shown increasing sleeping problems among pathologic gamblers, controlled for psychiatric comorbidity (Parhami et al., 2013). In accordance with other research, higher age was associated with prescription of hypnotic drugs in the group of patients studied here (Ancoli-Israel, 2009).

Antipsychotic drugs were prescribed to a total of $23.2 \%$ of the study participants, with a significant increase; the remarkable odds ratio of 4.39, over periods $(11.4-21.0 \%$ from period 1 to 3). In Sweden, antipsychotic drugs were dispensed to about $2 \%$ of the general population, a number appearing to be quite stable during the study period (Swedish National Board of Welfare, 2020b). Prevalence rates of psychotic disorders are reported to be about $4 \%$ in the general population, with a corresponding number of $7 \%$ in problem gamblers (Kim et al., 2018; Moreno-Kustner et al., 2018); hence, it is likely that many of the patients studied here were prescribed antipsychotic drugs on other indications than actual psychotic disorders. Increases of off-label prescription of atypical antipsychotic drugs for several psychiatric disorders have been reported, despite highly limited evidence (Albert et al., 2016). More attention needs to be drawn to the association between psychoses and GD, since this type of comorbidity may contribute to a higher gambling severity due to greater levels of impulsivity, and reward-directed behavioral disturbance requiring targeted treatment (Kim et al., 2018). Some dopamine antagonists themselves have been reported to, paradoxically as it may seem, increase the rewarding and priming effect of gambling in individuals with GD (Zack \& Poulos, 2007). Lithium is a mood-stabilizing and anti-impulsive drug with a relatively strict indication for bipolar disorder (The Swedish Association of the Pharmaceutical Industry 2020), a psychiatric condition characterized by alterations between depressive and manic episodes (American Psychiatric Association 2013) which can be closely linked to urges, reward-seeking behavior, and arousal in problem gamblers. Lithium has been suggested to affect certain regions of the brain which seem to be functionally impaired in both GD and bipolar disorder patients (Hollander et al., 2008), and a treatment algorithm with a dimensional approach has been presented by di Nicola and colleagues (2014). For participants in the current study, it is possible that symptoms of GD and bipolar disorder catalyzed each other, and prescription of lithium might has been beneficial for both diagnoses, but evidence is limited and more RCTs are required to evaluate the efficacy (Di Nicola et al., 2014). Similar to what we found out about the prescription of antiepileptics in this group of GD patients, the comorbidity based on prescription of antipsychotics is a non-specific but telling proxy for severe mental problems and suffering, here presented in connection with GD diagnoses.

Gambling research has been criticized for a bias toward men in treatment samples, but recently, more attention has been paid to gender questions in international studies and in Sweden specifically (Hakansson \& Widinghoff, 2020a; McCarthy et al., 2019). In the current setting, women have appeared as a group with increasing, rapidly progressing gambling problems and high psychiatric comorbidity (Hakansson \& Widinghoff, 2020b; Hakansson et al., 2017, 2018), in line with the well-known pathways model, where women are over-represented in the emotionally vulnerable group (Blaszczynski \& Nower, 2002; Valleur et al., 2016). The results of the present study further confirm this picture, with significantly higher prescription of drugs prescribed to treat depression, anxiety, and sleeping problems among women. Women in the general population are prescribed antidepressants twice as often as men (Swedish National Board of Welfare, 2019), and in our study, women 
had an OR of 5.2 (95\% CI 3.58-7.54, $p<0.001)$ compared to men. Another recent study illustrated this vulnerability, presenting that problem gambling in women was usually preceded by anxiety and/or depression, while men more often had gambling problems before any other psychiatric condition (Sundqvist \& Rosendahl, 2019). In the current study, there were no significant differences between genders concerning prescription of benzodiazepines, unlike what was seen in other drugs addressing anxiety and antidepressants. The addictive potential, use and misuse, and gender differences concerning benzodiazepines have previously been described. According to a comprehensive register study by Maust and colleagues (2019), benzodiazepines were prescribed more often to women, but with a significantly higher abuse in men. One possible interpretation of our results could be that men with gambling problems have more pronounced anxiety problems responding to benzodiazepine treatment compared to men in general, and there is also a marked risk of misuse in this group.

As other psychiatric disorders, gambling problems develop because of many different reasons, associated to both genetic and environmental factors (Gyollai et al., 2014). GD patients present psychiatric symptoms and cognitive deficits in a variety of domains overlapping with several other psychiatric disorders, and the underlying pathophysiological mechanisms, as well as the pharmacotherapeutic responses, are not fully understood (Di Nicola et al., 2014). Interpretation of the current study is complex, but the implications can be considered relevant both in a preventive - and treating context. Our results elucidate a group of patients probably experiencing severe suffering, as well as insufficient psychiatric care. This study indicates a generally worsened mental health for both men and women years prior to and following the GD diagnoses, despite on-going psychiatric medication, pointing out the need of optimized screening and treatment for GD and psychiatric comorbidity. In line with the systematic review and meta-analysis by Dowling and colleague (2015), our study indicates that it is of great importance to perform comprehensive assessment of co-occurring psychiatric disorders in GD patients, and by the method of the present study, we can also emphasize the need of GD screening, as a great majority of the patients were pharmacologically treated for other psychiatric conditions foregoing the GD diagnoses. Despite being able to unravel the actual chronology between GD and the psychiatric comorbidity in this study, our results highlight the severity of the situation for these patients, and implementation of systematic screening routines in specialized health care, or other institutions where patients with deteriorating mental health may seek help, could be a measure to reduce the stigma (Gainsbury et al., 2014) and discover problematic gambling at an earlier stage. Apart from the question of chronology between different psychiatric disorders, the importance of discovering gambling problems can probably not be overstated, as suicidal thoughts and attempts are associated with poor mental health, and markedly more common among people with financial problems and GD (Moghaddam et al., 2015; Swedish National Board of Welfare, 2019). This study, presenting a relatively distinct picture of the psychiatric comorbidity, also carries implications for clinical work with GD patients, as better knowledge and adequate handling of comorbidity is suggested being an important part of their treatment (Dowling et al., 2015a, 2015b). All types of psychiatric drugs were prescribed more commonly to the study participants compared to the general population. Our study shows that the increase in prescription of benzodiazepines, which are highly addictive, flattened out after establishment of GD diagnoses, while prescription of psychostimulants continued to increase in the corresponding situation. Besides the question of efficacy, safety is a highly important issue when considering benzodiazepines or psychostimulants to patients with addictive disorders, including GD, and more research is needed to rule out the most effective and safe treatment in a long-term perspective. Before 
initiating treatment with possibly addictive drugs to these patients, careful deliberation of risks of diversion and side effects versus therapeutic benefits should be made, and particular attention should be paid to the risks of deteriorated GD (Zack \& Poulos, 2004) or transition to substance abuse. To date, the opioid antagonist naltrexone is not approved for GD but may be the most promising pharmacological treatment option, especially for individuals with comorbid alcohol use disorder (Yip \& Potenza, 2014) and some of the prescription studied here may have occurred off-label addressing GD. Considering current knowledge, hypothetically, parts of the subgroup described here could have benefitted especially from treatment with naltrexone, but this is beyond the scope of this study and requires further research. Taken together, the results of this study as well as previous research in this area highlight the need of investigating possible psychiatric comorbidity in GD patients to assess appropriate treatment on an individual basis. GD is a heterogenic condition, involving highly complex biological pathways, and identifying subgroups based on psychiatric comorbidity could be part of the progression toward optimized pharmacological and behavioral therapy (Victorri-Vigneau et al., 2018). The overlapping comorbidity, here presented by the generally high prescription of psychiatric drugs, further points toward the complexity of the situation and could be looked at as a part of the debate raising the need of holistic perspective in psychiatric diagnostics, rather than maintenance of the current dichotomous system presuming distinct, discrete categories (Marshall, 2020). Considering the varying pathways and courses of GD, subgrouping could possibly form a basis for targeted measures (Di Nicola et al., 2014). There is a large need of RCTs and longitudinal studies evaluating psychosocial and pharmacological treatment in GD patients with respect to psychiatric comorbidity. Women were overrepresented in several of the investigated drug groups in this study, and better understanding of gender differences could be valuable to form preventive actions, and in situations of monitoring treatment plans and follow-up. Our results might contribute to development of evaluations of combined treatment strategies in large samples, based on the differences in comorbidity, which have been demanded (Lupi et al., 2014).

The present study has limitations, in part since psychiatric drugs are somewhat inexplicit proxies for psychiatric comorbidity, considering the wide range of indications, off label areas of use and general complexity in psychiatric diagnostics. The construct of a number of psychiatric diagnoses is questioned for their reliability (Kendell et al., 2003; Marshall, 2020; Plana-Ripoll et al., 2019), and the indications of most psychiatric drugs are highly varying (The Swedish Association of the Pharmaceutical Industry, 2020), making it difficult to draw conclusions from our study results in this area. Altogether though, we think that the overall picture of the described comorbidity in this study is in line with what could be expected from corresponding studies and populations (Dowling et al., 2015b; Hakansson et al., 2018). Another limiting factor in the current methodology is the lack of temporal precision of GD. Definitionally, period 3 started on the day GD diagnoses were clinically established but most likely, varying or escalating gambling problems had been present before that time. Accordingly, we cannot claim what happened in detail before or after the initiation of gambling problems, but rather describe a wider picture of the mental health during a period when GD was observed in specialized health care. Neither can we state whether changes in prescription following GD diagnoses are influenced by establishment of the GD diagnoses, by establishment of other diagnoses which were made during the same period, or both. The selection of participants emanates from patients who had received their GD diagnoses in specialized health care, consequently affecting the generalizability of the results. This group of patients can be presumed to have suffered from relatively severe GD and psychiatric comorbidity; however, also community samples 
of problem gamblers have been presenting with considerable levels of psychiatric comorbidity (Lorains et al., 2011). This study is a description of psychiatric drugs prescribed in connections with establishment of GD diagnoses but does not investigate the possible pharmacological effects on gambling problems, nor psychiatric symptoms of any kind. The conclusion that the studied group displays an extensive and increasing mental distress is thus an interpretation of the signification of psychiatric drug prescription, and not a measure of actual psychiatric disorders or symptoms, except for the GD diagnoses.

\section{Conclusions}

This study describes the mental health in a large clinical GD sample, by using national register data on GD diagnoses and prescribed drugs. Our results indicate extensive psychiatric comorbidity and deteriorated mental health prior to, and following, establishment of GD diagnoses, despite extensive pharmacological treatment. The prescription of all investigated drug groups increased significantly over the three studied periods, except for psychostimulants. After GD diagnoses had been established, prescription of benzodiazepines flattened out, while prescription of psychostimulants increased significantly. Women had a significantly higher prescription of antiepileptics, anxiolytics, hypnotics, and antidepressants, pointing toward a higher emotional vulnerability in this group. The results call for further efforts to screen, and offering treatment, for GD in groups with mental health problems. Optimizing psychosocial and pharmacotherapeutic interventions for GD in patients with psychiatric comorbidity, and vice versa, at an early stage, is probably an important key to successful treatment. Further studies could aim to find suitable treatment algorithms, addressing individual needs (Di Nicola et al., 2014). More research is needed to optimize targeted treatment for patients suffering from GD and psychiatric comorbidity.

Funding Open access funding provided by Lund University.

\section{Declarations}

Conflict of Interest Carolina Widinghoff holds a researcher position at Lund University, Sweden, in collaboration with the Swedish state-owned gambling monopoly, Svenska Spel AB. She also has a collaboration with Kontigo Care, a private company, in a clinical study on technical follow-up tools in clinical treatment, where the devices to be studied are to be provided free of charge, but with no direct financial support from that company. None of the bodies listed above had any role in or influence on the present project.

Jonas Berge declares that he has no conflict of interest.

Hakansson's position as a professor at Lund University is financially supported by the Swedish state-owned gambling operator AB Svenska Spel. He also has applied for and obtained research funding from the research council of the same company, as well as from the research council of Systembolaget AB, the Swedish state-owned alcohol monopoly, and the research council of the Swedish sports federation and the Swedish enforcement agency. He also has a collaboration with Kontigo Care, a private company, in a clinical study on technical follow-up tools in clinical treatment, where the devices to be studied are to be provided free of charge, but with no direct financial support from that company. None of the bodies listed above had any role in or influence on the present project.

Open Access This article is licensed under a Creative Commons Attribution 4.0 International License, which permits use, sharing, adaptation, distribution and reproduction in any medium or format, as long as you give appropriate credit to the original author(s) and the source, provide a link to the Creative Commons licence, and indicate if changes were made. The images or other third party material in this article are included in the article's Creative Commons licence, unless indicated otherwise in a credit line to the 
material. If material is not included in the article's Creative Commons licence and your intended use is not permitted by statutory regulation or exceeds the permitted use, you will need to obtain permission directly from the copyright holder. To view a copy of this licence, visit http://creativecommons.org/licenses/by/4.0/.

\section{References}

Abbott, M., Romild, U., \& Volberg, R. (2017). The prevalence, incidence, and gender and age-specific incidence of problem gambling: results of the Swedish longitudinal gambling study (Swelogs). Addiction. https://doi.org/10.1111/add.14083

Abbott, M., Romild, U., \& Volberg, R. (2018). The prevalence, incidence, and gender and age-specific incidence of problem gambling: results of the Swedish longitudinal gambling study (Swelogs). Addiction, 113(4), 699-707. https://doi.org/10.1111/add.14083

Albert, U., Carmassi, C., Cosci, F., De Cori, D., Di Nicola, M., Ferrari, S., Poloni, N., Tarricone, I., \& Fiorillo, A. (2016). Role and clinical implications of atypical antipsychotics in anxiety disorders, obsessive-compulsive disorder, trauma-related, and somatic symptom disorders: a systematized review. Int Clin Psychopharmacol, 31(5), 249-258. https://doi.org/10.1097/YIC.0000000000 000127

American Psychiatric Association (2013). Diagnostic and statistical manual of psychiatric disorders (5th ed. ed.). American Psychiatric Publishing.

Ancoli-Israel, S. (2009). Sleep and its disorders in aging populations. Sleep Med, 10(Suppl 1), S7-11. https://doi.org/10.1016/j.sleep.2009.07.004

Balon, R. (2013). Benzodiazepines revisited. Psychotherapy and Psychosomatics, 82(6), 353-354. https://doi.org/10.1159/000353599

Biederman, J. (2003). Pharmacotherapy for attention-deficit/hyperactivity disorder (ADHD) decreases the risk for substance abuse: findings from a longitudinal follow-up of youths with and without ADHD. J Clin Psychiatry, 64 Suppl 11,3-8. https://www.ncbi.nlm.nih.gov/pubmed/14529323

Blaszczynski, A., \& Nower, L. (2002). A pathways model of problem and pathological gambling. Addiction, 97(5), 487-499. https://www.ncbi.nlm.nih.gov/pubmed/12033650

Chamberlain, S. R., \& Grant, J. E. (2019). Efficacy of pharmacological interventions in targeting decision-making impairments across substance and behavioral addictions. Neuropsychol Rev, 29(1), 93-102. https://doi.org/10.1007/s11065-019-09400-z

Chang, Z., Lichtenstein, P., Halldner, L., D’Onofrio, B., Serlachius, E., Fazel, S., Langstrom, N., \& Larsson, H. (2014). Stimulant ADHD medication and risk for substance abuse. J Child Psychol Psychiatry, 55(8), 878-885. https://doi.org/10.1111/jcpp.12164

Clemow, D. B. (2017). Misuse of methylphenidate. Current Topics in Behavioral Neurosciences, 34, 99-124. https://doi.org/10.1007/7854_2015_426

Crunelle, C. L., van den Brink, W., Moggi, F., Konstenius, M., Franck, J., Levin, F. R., van de Glind, G., Demetrovics, Z., Coetzee, C., Luderer, M., Schellekens, A., Group, I. C., \& Matthys, F. (2018). International Consensus Statement on Screening, Diagnosis and Treatment of Substance Use Disorder Patients with Comorbid Attention Deficit/Hyperactivity Disorder. Eur Addict Res, 24(1), 43-51. https://doi.org/10.1159/000487767

Cunill, R., Castells, X., Tobias, A., \& Capella, D. (2015). Pharmacological treatment of attention deficit hyperactivity disorder with co-morbid drug dependence. J Psychopharmacol, 29(1), 15-23. https:// doi.org/10.1177/0269881114544777

Dell'Osso, B., Albert, U., Atti, A. R., Carmassi, C., Carra, G., Cosci, F., Del Vecchio, V., Di Nicola, M., Ferrari, S., Goracci, A., Iasevoli, F., Luciano, M., Martinotti, G., Nanni, M. G., Nivoli, A., Pinna, F., Poloni, N., Pompili, M., Sampogna, G., ... Fiorillo, A. (2015). Bridging the gap between education and appropriate use of benzodiazepines in psychiatric clinical practice. Neuropsychiatric Disease and Treatment, 11, 1885-1909. https://doi.org/10.2147/NDT.S83130

Di Nicola, M., De Crescenzo, F., D’Alo, G. L., Remondi, C., Panaccione, I., Moccia, L., Molinaro, M., Dattoli, L., Lauriola, A., Martinelli, S., Giuseppin, G., Maisto, F., Crosta, M. L., Di Pietro, S., Amato, L., \& Janiri, L. (2020). Pharmacological and psychosocial treatment of adults with gambling disorder: a meta-review. J Addict Med, 14(4), e15-e23. https://doi.org/10.1097/ADM.00000 00000000574

Di Nicola, M., De Risio, L., Pettorruso, M., Caselli, G., De Crescenzo, F., Swierkosz-Lenart, K., Martinotti, G., Camardese, G., Di Giannantonio, M., \& Janiri, L. (2014). Bipolar disorder and gambling 
disorder comorbidity: Current evidence and implications for pharmacological treatment. Journal of Affective Disorders, 167, 285-298. https://doi.org/10.1016/j.jad.2014.06.023

Di Nicola, M., Pepe, M., Modica, M., Lanzotti, P., Panaccione, I., Moccia, L., \& Janiri, L. (2020). Mixed states in patients with substance and behavioral addictions. Psychiatr Clin North Am, 43(1), 127137. https://doi.org/10.1016/j.psc.2019.10.012

Dowling, N. A., Cowlishaw, S., Jackson, A. C., Merkouris, S. S., Francis, K. L., \& Christensen, D. R. (2015). The prevalence of comorbid personality disorders in treatment-seeking problem gamblers: a systematic review and meta-analysis. J Pers Disord, 29(6), 735-754. https://doi.org/10.1521/ pedi_2014_28_168

Dowling, N. A., Cowlishaw, S., Jackson, A. C., Merkouris, S. S., Francis, K. L., \& Christensen, D. R. (2015). Prevalence of psychiatric co-morbidity in treatment-seeking problem gamblers: a systematic review and meta-analysis. Aust N Z J Psychiatry, 49(6), 519-539. https://doi.org/10.1177/ 0004867415575774

Ford, M., \& Hakansson, A. (2020). Problem gambling, associations with comorbid health conditions, substance use, and behavioural addictions: Opportunities for pathways to treatment. PLoS ONE, 15(1), e0227644. https://doi.org/10.1371/journal.pone.0227644

Gainsbury, S., Hing, N., \& Suhonen, N. (2014). Professional help-seeking for gambling problems: awareness, barriers and motivators for treatment. J Gambl Stud, 30(2), 503-519. https://doi.org/10.1007/ s10899-013-9373-X

Goodman, C. W., \& Brett, A. S. (2017). Gabapentin and pregabalin for pain - is increased prescribing a cause for concern? N Engl J Med, 377(5), 411-414. https://doi.org/10.1056/NEJMp1704633

Goslar, M., Leibetseder, M., Muench, H. M., Hofmann, S. G., \& Laireiter, A. R. (2019). Pharmacological treatments for disordered gambling: a meta-analysis. J Gambl Stud, 35(2), 415-445. https://doi.org/10. 1007/s 10899-018-09815-y

Gyollai, A., Griffiths, M. D., Barta, C., Vereczkei, A., Urban, R., Kun, B., Kokonyei, G., Szekely, A., Sasvari-Szekely, M., Blum, K., \& Demetrovics, Z. (2014). The genetics of problem and pathological gambling: A systematic review. Current Pharmaceutical Design, 20(25), 3993-3999. https://doi.org/10. 2174/13816128113199990626

Hakansson, A., Karlsson, A., \& Widinghoff, C. (2018). Primary and secondary diagnoses of gambling disorder and psychiatric comorbidity in the Swedish health care system-a nationwide register study. Front Psychiatry, 9, 426. https://doi.org/10.3389/fpsyt.2018.00426

Hakansson, A., Mardhed, E., \& Zaar, M. (2017). Who seeks treatment when medicine opens the door to pathological gambling patients-psychiatric comorbidity and heavy predominance of online gambling. Front Psychiatry, 8, 255. https://doi.org/10.3389/fpsyt.2017.00255

Hakansson, A., \& Widinghoff, C. (2020a). Gender differences in problem gamblers in an online gambling setting. Psychology Research and Behavior Management, 13, 681-691. https://doi.org/10.2147/PRBM. S248540

Hakansson, A., \& Widinghoff, C. (2020b). Over-indebtedness and problem gambling in a general population sample of online gamblers. Front Psychiatry, 11, 7. https://doi.org/10.3389/fpsyt.2020.00007

Hellberg, S. N., Russell, T. I., \& Robinson, M. J. F. (2019). Cued for risk: evidence for an incentive sensitization framework to explain the interplay between stress and anxiety, substance abuse, and reward uncertainty in disordered gambling behavior. Cogn Affect Behav Neurosci, 19(3), 737-758. https://doi. org/10.3758/s13415-018-00662-3

Hollander, E., Buchsbaum, M. S., Haznedar, M. M., Berenguer, J., Berlin, H. A., Chaplin, W., Goodman, C. R., LiCalzi, E. M., Newmark, R., \& Pallanti, S. (2008). FDG-PET study in pathological gamblers 1 Lithium increases orbitofrontal, dorsolateral and cingulate metabolism. Neuropsychobiology, 58(1), 37-47. https://doi.org/10.1159/000154478

Humphreys, K. L., Eng, T., \& Lee, S. S. (2013). Stimulant medication and substance use outcomes: a metaanalysis. JAMA Psychiatry, 70(7), 740-749. https://doi.org/10.1001/jamapsychiatry.2013.1273

IBM Corp. (2019) IBM SPSS Statistics for Windows, Version 26.0.

Kendell, R., \& Jablensky, A. (2003). Distinguishing between the validity and utility of psychiatric diagnoses. Am J Psychiatry, 160(1), 4-12. https://doi.org/10.1176/appi.ajp.160.1.4

Kim, H. S., Cassetta, B. D., Hodgins, D. C., Tomfohr-Madsen, L. M., McGrath, D. S., \& Tavares, H. (2018). Assessing the relationship between disordered gamblers with psychosis and increased gambling severity: the mediating role of impulsivity. Can J Psychiatry, 63(6), 370-377. https://doi.org/10.1177/07067 43717730825

Kurko, T., Saastamoinen, L. K., Tuulio-Henriksson, A., Taiminen, T., Tiihonen, J., Airaksinen, M., \& Hietala, J. (2018). Trends in the long-term use of benzodiazepine anxiolytics and hypnotics: a national register study for 2006 to 2014. Pharmacoepidemiol Drug Saf, 27(6), 674-682. https://doi.org/10. $1002 /$ pds.4551 
LaPlante, D. A., Nelson, S. E., LaBrie, R. A., \& Shaffer, H. J. (2008). Stability and progression of disordered gambling: lessons from longitudinal studies. Can J Psychiatry, 53(1), 52-60. https://doi.org/10. 1177/070674370805300108

LiverTox: Clinical and research information on drug-induced liver injury [Internet].

Bethesda (MD): National Institute of Diabetes and Digestive and Kidney Diseases; 2012-. Hydroxyzine. 2017.

Lorains, F. K., Cowlishaw, S., \& Thomas, S. A. (2011). Prevalence of comorbid disorders in problem and pathological gambling: systematic review and meta-analysis of population surveys. Addiction, 106(3), 490-498. https://doi.org/10.1111/j.1360-0443.2010.03300.x

Ludvigsson, J. F., Andersson, E., Ekbom, A., Feychting, M., Kim, J. L., Reuterwall, C., Heurgren, M., \& Olausson, P. O. (2011). External review and validation of the Swedish national inpatient register. $B M C$ Public Health, 11, 450. https://doi.org/10.1186/1471-2458-11-450

Lupi, M., Martinotti, G., Acciavatti, T., Pettorruso, M., Brunetti, M., Santacroce, R., Cinosi, E., Di Iorio, G., Di Nicola, M., \& Di Giannantonio, M. (2014). Pharmacological treatments in gambling disorder: A qualitative review. BioMed Research International, 2014, 537306. https://doi.org/10.1155/ 2014/537306

Maglione, M., Maher, A. R., Hu, J., Wang, Z., Shanman, R., Shekelle, P. G., Roth, B., Hilton, L., Suttorp, M. J., Ewing, B. A., Motala, A., \& Perry, T. (2011). In off-label use of atypical antipsychotics: an update. https://www.ncbi.nlm.nih.gov/pubmed/22132426

Marshall, M. (2020, May). The hidden links between mental disorders. Nature, 581(7806), 19-21. https://doi.org/10.1038/d41586-020-00922-8

McCarthy, S., Thomas, S. L., Bellringer, M. E., \& Cassidy, R. (2019). Women and gambling-related harm: a narrative literature review and implications for research, policy, and practice. Harm Reduct J, 16(1), 18. https://doi.org/10.1186/s12954-019-0284-8

Mitchell, M. R., \& Potenza, M. N. (2014). Addictions and personality traits: impulsivity and related constructs. Curr Behav Neurosci Rep, 1(1), 1-12. https://doi.org/10.1007/s40473-013-0001-y

Moghaddam, J. F., Yoon, G., Dickerson, D. L., Kim, S. W., \& Westermeyer, J. (2015). Suicidal ideation and suicide attempts in five groups with different severities of gambling: findings from the National Epidemiologic Survey on Alcohol and Related Conditions. Am J Addict, 24(4), 292-298. https:// doi.org/10.1111/ajad.12197

Moreno-Kustner, B., Martin, C., \& Pastor, L. (2018). Prevalence of psychotic disorders and its association with methodological issues. A systematic review and meta-analyses. PLoS One, 13(4), e0195687. https://doi.org/10.1371/journal.pone.0195687

Muller, K. W., Wolfling, K., Dickenhorst, U., Beutel, M. E., Medenwaldt, J., \& Koch, A. (2017). Recovery, relapse, or else? Treatment outcomes in gambling disorder from a multicenter follow-up study. European Psychiatry, 43, 28-34. https://doi.org/10.1016/j.eurpsy.2017.01.326

Naguy, A., \& Al-Enezi, N. (2019). Lamotrigine uses in psychiatric practice. Am J Ther, 26(1), e96-e102. https://doi.org/10.1097/MJT.0000000000000535

Parhami, I., Siani, A., Rosenthal, R. J., \& Fong, T. W. (2013). Pathological gambling, problem gambling and sleep complaints: an analysis of the National Comorbidity Survey: Replication (NCS-R). J Gambl Stud, 29(2), 241-253. https://doi.org/10.1007/s10899-012-9299-8

Perugi, G., Pallucchini, A., Rizzato, S., De Rossi, P., Sani, G., Maremmani, A. G., Pinzone, V., \& Maremmani, I. (2019). Pharmacotherapeutic strategies for the treatment of attention-deficit hyperactivity (ADHD) disorder with comorbid substance-use disorder (SUD). Expert Opin Pharmacother, 20(3), 343-355. https://doi.org/10.1080/14656566.2018.1551878

Petry, N. M., Stinson, F. S., \& Grant, B. F. (2005). Comorbidity of DSM-IV pathological gambling and other psychiatric disorders: results from the National Epidemiologic Survey on Alcohol and Related Conditions. J Clin Psychiatry, 66(5), 564-574. https://www.ncbi.nlm.nih.gov/pubmed/ 15889941

Plana-Ripoll, O., Pedersen, C. B., Holtz, Y., Benros, M. E., Dalsgaard, S., de Jonge, P., Fan, C. C., Degenhardt, L., Ganna, A., Greve, A. N., Gunn, J., Iburg, K. M., Kessing, L. V., Lee, B. K., Lim, C. C. W., Mors, O., Nordentoft, M., Prior, A., Roest, A. M., ... McGrath, J. J. (2019). Exploring comorbidity within mental disorders among a Danish national population. JAMA Psychiatry, 76(3), 259-270. https://doi.org/10.1001/jamapsychiatry.2018.3658

Proctor, A., \& Bianchi, M. T. (2012). Clinical pharmacology in sleep medicine. ISRN Pharmacol, 2012, 914168. https://doi.org/10.5402/2012/914168

R Core Team (2019). A language and environment for statistical computing. R Foundation for Statistical Computing. https://www.R-project.org/ 
Ronzitti, S., Soldini, E., Lutri, V., Smith, N., Clerici, M., \& Bowden-Jones, H. (2016). Types of gambling and levels of harm: a UK study to assess severity of presentation in a treatment-seeking population. J Behav Addict, 5(3), 439-447. https://doi.org/10.1556/2006.5.2016.068

Setlik, J., Bond, G. R., \& Ho, M. (2009). Adolescent prescription ADHD medication abuse is rising along with prescriptions for these medications. Pediatrics, 124(3), 875-880. https://doi.org/10. 1542/peds.2008-0931

Slutske, W. S. (2006). Natural recovery and treatment-seeking in pathological gambling: results of two U.S. national surveys. Am J Psychiatry, 163(2), 297-302. https://doi.org/10.1176/appi.ajp.163.2.297

Sundqvist, K., \& Rosendahl, I. (2019). Problem gambling and psychiatric comorbidity-risk and temporal sequencing among women and men: results from the Swelogs case-control study. J Gambl Stud, 35(3), 757-771. https://doi.org/10.1007/s10899-019-09851-2

Suurvali, H., Cordingley, J., Hodgins, D. C., \& Cunningham, J. (2009). Barriers to seeking help for gambling problems: a review of the empirical literature. J Gambl Stud, 25(3), 407-424. https://doi.org/ 10.1007/s10899-009-9129-9

Swedish Medical Products Agency (2016). Treatment recommendations for ADHD. Report number 2:2016. Retrieved 2020, from https://www.lakemedelsverket.se/4a4255/globalassets/dokument/behandlingoch-forskrivning/behandlingsrekommendationer/behandlingsrekommendation/behandlingsrekommend ation-lakemedel-adhd.pdf

Swedish National Board of Welfare (2018). Drug statistics 2017. https://www.socialstyrelsen.se/globalasse ts/sharepoint-dokument/artikelkatalog/statistik/2018-4-7.pdf

Swedish National Board of Welfare (2019). Vård vid depression och ångestsyndrom 2019 Underlagsrapport. https://www.socialstyrelsen.se/globalassets/sharepoint-dokument/artikelkatalog/nationella-riktl injer/2019-5-13.pdf

Swedish National Board of Welfare (2020a). Information för Patientregistret, registerår 2018, version 5. https://www.socialstyrelsen.se/globalassets/sharepoint-dokument/dokument-webb/statistik/infor mation-for-patientregistret-registerar-2018.pdf

Swedish National Board of Welfare (2020b). Statistic Database of National Drug Prescription Retrieved 20th of June from https://sdb.socialstyrelsen.se/if_lak/val.aspx

The Swedish Association of the Pharmaceutical Industry (2020). FASS. Farmaceutiska specialiteter i Sverige. The Swedish Association of the Pharmaceutical Industry, Pharmaceutic specialities in Sweden. Retrieved 2020.09.22, from www.fass.se

Torres-Bondia, F., de Batlle, J., Galvan, L., Buti, M., Barbe, F., \& Pinol-Ripoll, G. (2020). Trends in the consumption rates of benzodiazepines and benzodiazepine-related drugs in the health region of Lleida from 2002 to 2015. BMC Public Health, 20(1), 818. https://doi.org/10.1186/s12889-020-08984-z

Valleur, M., Codina, I., Venisse, J. L., Romo, L., Magalon, D., Fatseas, M., Chereau-Boudet, I., Gorsane, M. A., Guilleux, A., Groupe, J. E. U., Grall-Bronnec, M., \& Challet-Bouju, G. (2016). Towards a validation of the three pathways model of pathological gambling. J Gambl Stud, 32(2), 757-771. https://doi. org/10.1007/s10899-015-9545-y

Victorri-Vigneau, C., Spiers, A., Caillet, P., Bruneau, M., Ignace, C., Challet-Bouju, G., \& Grall-Bronnec, M. (2018). Opioid antagonists for pharmacological treatment of gambling disorder: Are they relevant? Current Neuropharmacology, 16(10), 1418-1432. https://doi.org/10.2174/1570159X156661707181 44058

Wardle, H., Reith, G., Langham, E., \& Rogers, R. D. (2019). Gambling and public health: we need policy action to prevent harm. BMJ, 365, 1807. https://doi.org/10.1136/bmj.11807

Wick, J. Y. (2013). The history of benzodiazepines. Consult Pharm, 28(9), 538-548. https://doi.org/10. 4140/TCP.n.2013.538

World Health Organization (2004). ICD-10: international statistical classification of diseases and related health problems: tenth revision, 2nd ed." World Health Organization. https://apps.who.int/iris/handle/ $10665 / 42980$

World Health Organization (2020). Anatomical therapeutic chemical classification system. ATC Index 2020. World Health Organization. Retrieved October from https://www.whocc.no/atc_ddd_index/

Yip, S. W., \& Potenza, M. N. (2014). Treatment of gambling disorders. Curr Treat Options Psychiatry, 1(2), 189-203. https://doi.org/10.1007/s40501-014-0014-5

Zack, M., \& Poulos, C. X. (2004). Amphetamine primes motivation to gamble and gambling-related semantic networks in problem gamblers. Neuropsychopharmacology, 29(1), 195-207. https://doi.org/10. 1038/sj.npp.1300333

Zack, M., \& Poulos, C. X. (2007). A D2 antagonist enhances the rewarding and priming effects of a gambling episode in pathological gamblers. Neuropsychopharmacology, 32(8), 1678-1686. https://doi.org/ 10.1038/sj.npp.1301295 
Zack, M., \& Poulos, C. X. (2009). Parallel roles for dopamine in pathological gambling and psychostimulant addiction. Curr Drug Abuse Rev, 2(1), 11-25. https://doi.org/10.2174/1874473710902010011

Publisher's Note Springer Nature remains neutral with regard to jurisdictional claims in published maps and institutional affiliations. 